\title{
Urinary angiotensinogen as a marker of elevated blood pressure in patients with chronic kidney disease
}

\author{
Chan-Young Jung and Tae-Hyun Yoo
}

Department of Internal Medicine, Institute of Kidney Disease Research, Yonsei University College of Medicine, Seoul, Korea

Received: April 9, 2021

Accepted: April 15, 2021

\section{Correspondence to}

Tae-Hyun Yoo, M.D.

Department of Internal

Medicine, Institute of Kidney

Disease Research, Yonsei University College of Medicine, 50 Yonsei-ro, Seodaemun-gu, Seoul 03722, Korea

Tel: +82-2-2228-1975

Fax: +82-2-393-6884

E-mail: yoosyo316@yuhs.ac https://orcid.org/0000-00029183-4507

\section{See Article on Page 659-667}

The renin-angiotensin system (RAS) is one of the most important systems for blood pressure regulation and sodium homeostasis in patients with chronic kidney disease (CKD). As well as systemic RAS activity, the role of the local RAS in specific tissues and organs, including the kidneys, has been the focus of many studies. All three components of the RAS co-exist in the kidneys. These components are locally activated and play a role in the development and progression of various kidney diseases [1]. The RAS is the target of many antihypertensive drugs due to its important role in blood pressure regulation. However, inappropriate activation of the intrarenal RAS due to arteriosclerotic narrowing of the renal arterioles is a major contributor to excess sodium retention, and an important mechanism underlying elevated blood pressure and progression of CKD [2].

Angiotensinogen (AGT) is the only known substrate for renin, which is the rate-determining enzyme of the RAS. Therefore, changes in AGT or renin levels affect RAS activity, as evidenced by an in vitro study [3]. Kobori et al. [4] revealed that urinary angiotensinogen (UAGT) levels are correlated with intrarenal AGT and angiotensin II levels in angiotensin II-induced and spontaneously hypertensive rats. It is unknown whether UAGT, which is largely synthesized in the proximal kidney tubules, can be used as a marker of intrarenal RAS activity in patients with CKD. However, Yamamoto et al. [5] demonstrated that high UAGT levels are present in patients with a low estimated glomerular filtration rate and high urinary protein excretion rate, and that treatment with the angiotensin receptor blocker losartan reduces UAGT levels.

As previously mentioned, the RAS also plays an important role in sodium homeostasis. Several epidemiological studies have reported that the prevalence of elevated blood pressure is directly related to dietary salt intake in all populations with a sodium intake of 50 to $100 \mathrm{mmol} /$ day $[6,7]$, whereas the prevalence of hypertension is rare in populations with low salt intake.

Salt loading usually induces urinary salt excretion, called pressure natriuresis; however, some individuals have a blunted response to high salt intake. Salt sensitivity describes individuals whose blood pressure decreases and increases during periods of salt restriction and loading, respectively. Risk fac- 
tors for salt sensitivity include old age, obesity, diabetes, and CKD. Although the association between salt sensitivity and an increased risk of hypertension and cardiovascular diseases is clear, the underlying pathogenic mechanism is not fully understood.

Considering that both activation of the intrarenal RAS and salt sensitivity contribute to elevated blood pressure, we postulate that there is a relationship between intrarenal RAS activity and salt sensitivity. However, evidence supporting this association has been conflicting. In a population-based dietary feeding study of 100 randomly selected participants, Rebholz et al. [8] demonstrated the relationship between salt sensitivity and intrarenal RAS activity, indexed by UAGT. However, the study was conducted on individuals without kidney disease. Kim et al. [9] investigated this association in a study of 1,955 patients with CKD. The association between UAGT excretion and elevated blood pressure, as well as its relationship with intra-renal RAS activity and dietary sodium, was determined. The study subjects were from the Korean Cohort Study for Outcome in Patients with Chronic Kidney Disease (KNOW-CKD) study, which is a patient-based multicenter cohort study involving nine tertiary care hospitals in South Korea. The authors reported that enhanced intrarenal RAS activity, as indicated by a high urinary angiotensinogen-to-creatinine ratio (UAGT/Cr), and high urinary sodium (UNa) excretion, as indicated by a high 24-hour UNa, were both associated with high systolic blood pressure in patients with CKD. The group in the highest UAGT/Cr and 24hour UNa tertile were also in the highest systolic blood pressure tertile, with an odds ratio of 2.4 compared to the rest of the group. Systolic blood pressure increased by $2.0 \mathrm{mmHg}$ for every $100 \mathrm{mEq} /$ day increase in 24 -hour $\mathrm{UNa}(p=0.027)$, which is a smaller change than reported in a study based on the Korean National Health and $\mathrm{Nu}$ tritional Examination Survey, where a $100 \mathrm{mEq} /$ day increase in 24-hour UNa was associated with a $6.1 \mathrm{mmHg}$ increase in systolic blood pressure. This further supports the concept of salt sensitivity, which could differ between individuals with and without CKD. An interesting finding from the study was that there was no correlation between UAGT excretion and UNa excretion, which contrasts with previous studies indicating that high salt intake leads to an increase in intrarenal RAS activity in salt-sensitive patients [10]. The study by Kim et al. [9] had several limitations. First, due to its cross-sectional nature, causal relationships between blood pressure, 24-hour UNa, and UAGT/Cr were difficult to determine. Second, a subgroup analysis of salt-sensitive and salt-resistant groups would have provided further insight into how UAGT, UNa excretion, and salt sensitivity interact in patients with CKD. Finally, it would have been interesting to see how enhanced intrarenal RAS activity affected long-term clinical outcomes, such as cardiovascular and kidney outcomes, in patients with CKD. Nonetheless, the study represents a leap forward in our understanding of a relatively novel biomarker and holds considerable promise to improve the quality of care for patients with CKD.

\section{Conflict of interest}

No potential conflict of interest relevant to this article was reported.

\section{REFERENCES}

1. Yoo TH, Li JJ, Kim JJ, et al. Activation of the renin-angiotensin system within podocytes in diabetes. Kidney Int 2007;71:1019-1027.

2. Kobori H, Nishiyama A, Abe Y, Navar LG. Enhancement of intrarenal angiotensinogen in Dahl salt-sensitive rats on high salt diet. Hypertension 2003;41:592-597.

3. Klett C, Nobiling R, Gierschik P, Hackenthal E. Angiotensin II stimulates the synthesis of angiotensinogen in hepatocytes by inhibiting adenylylcyclase activity and stabilizing angiotensinogen mRNA. J Biol Chem 1993;268:25095-25107.

4. Kobori H, Ozawa Y, Suzaki Y, Nishiyama A. Enhanced intrarenal angiotensinogen contributes to early renal injury in spontaneously hypertensive rats. J Am Soc Nephrol 2005;16:2073-2080.

5. Yamamoto T, Nakagawa T, Suzuki H, et al. Urinary angiotensinogen as a marker of intrarenal angiotensin II activity associated with deterioration of renal function in patients with chronic kidney disease. J Am Soc Nephrol 2007;18:1558-1565.

6. MacGregor GA. Sodium is more important than calcium in essential hypertension. Hypertension 1985;7:628-640.

7. Frame AA, Wainford RD. Renal sodium handling and sodium sensitivity. Kidney Res Clin Pract 2017;36:117-131. 
8. Rebholz CM, Chen J, Zhao Q, et al. Urine angiotensinogen and salt-sensitivity and potassium-sensitivity of blood pressure. J Hypertens 2015;33:1394-1400.

9. Kim HY, Choi HS, Kim CS, et al. Effect of urinary angiotensinogen and high-salt diet on blood pressure in patients with chronic kidney disease: results from the
Korean Cohort Study for Outcome in Patients with Chronic Kidney Disease (KNOW-CKD). Korean J Intern Med 2021;36:659-667.

10. Majid DS, Prieto MC, Navar LG. Salt-sensitive hypertension: perspectives on intrarenal mechanisms. Curr Hypertens Rev 2015;11:38-48. 